Technical Note

\title{
Extracting the Tailings Ponds from High Spatial Resolution Remote Sensing Images by Integrating a Deep Learning-Based Model
}

\author{
Jianjun Lyu ${ }^{1}$, Ying Hu ${ }^{1}$, Shuliang Ren ${ }^{1}$, Yao Yao ${ }^{1,2, * \mathbb{C}}$, Dan Ding ${ }^{3}$, Qingfeng Guan ${ }^{1} \mathbb{D}$ and Liufeng Tao ${ }^{4}$ \\ 1 School of Geography and Information Engineering, China University of Geoscience, Wuhan 430078, China; \\ jjlv@cug.edu.cn (J.L.); hhying@cug.edu.cn (Y.H.); renshuliang@cug.edu.cn (S.R.); guanqf@cug.edu.cn (Q.G.) \\ 2 Alibaba Group, Hangzhou 311121, China \\ 3 No.321 Geological Team, Bureau of Geological and Mineral Exploration of Anhui Province, \\ Tongling 244033, China; dingdan@ahdk321.com \\ 4 National Engineering Research Center for Geographic Information System, China University of Geoscience, \\ Wuhan 430078, China; taoliufeng@cug.edu.cn \\ * Correspondence: yaoy@cug.edu.cn; Tel.: +86-185-2023-9106
}

check for updates

Citation: Lyu, J.; Hu, Y.; Ren, S.; Yao, Y.; Ding, D.; Guan, Q.; Tao, L. Extracting the Tailings Ponds from High Spatial Resolution Remote Sensing Images by Integrating a Deep Learning-Based Model. Remote Sens. 2021, 13, 743. https://doi.org/ $10.3390 /$ rs13040743

Academic Editor: Sawaid Abbas

Received: 4 January 2021

Accepted: 15 February 2021

Published: 17 February 2021

Publisher's Note: MDPI stays neutral with regard to jurisdictional claims in published maps and institutional affiliations.

Copyright: (c) 2021 by the authors. Licensee MDPI, Basel, Switzerland. This article is an open access article distributed under the terms and conditions of the Creative Commons Attribution (CC BY) license (https:/ / creativecommons.org/licenses/by/ $4.0 /)$.

\begin{abstract}
Due to a lack of data and practical models, few studies have extracted tailings pond margins in large areas. In addition, there is no public dataset of tailings ponds available for relevant research. This study proposed a new deep learning-based framework for extracting tailings pond margins from high spatial resolution (HSR) remote sensing images by combining You Only Look Once (YOLO) v4 and the random forest algorithm. At the same time, we created an open source tailings pond dataset based on HSR remote sensing images. Taking Tongling city as the study area, the proposed model can detect tailings pond locations with high accuracy and efficiency from a large HSR remote sensing image $($ precision $=99.6 \%$, recall $=89.9 \%$, mean average precision $=89.7 \%$ ). An optimal random forest model and morphological processing were utilized to further extract accurate tailings pond margins from the target areas. The final map of the entire study area was obtained with high accuracy. Compared with the random forest algorithm, the total extraction time was reduced by nearly $99 \%$. This study can be beneficial to mine monitoring and ecological environmental governance.
\end{abstract}

Keywords: tailings ponds; remote sensing image; deep learning; random forest; high spatial resolution

\section{Introduction}

A tailings pond is a mine production facility used to store tailings generated during processing metal resources [1]. The liquid contained in tailings ponds is poisonous, harmful or radioactive [2]. The tailings pond usually requires a large area to contain all the produced tailings [3]. Restricted by mineral resources, terrain and other factors, tailings ponds are mostly located in remote mountainous areas with relatively weak supervision [4]. According to statistics, the number of known tailings ponds in China in 2019 was 5189. Most of them were distributed in isolation and nearly $80 \%$ of tailing ponds are less than $0.363 \mathrm{~km}^{2}$ in area [5]. Most tailings ponds use the upstream raising method due to the low operating costs. However, this method lacks stability. If a tailings dam breaks, it can cause severe casualties, economic losses and irreparable environmental pollution [6]. In recent years, tailings ponds have frequently caused environmental emergencies, which have had extremely adverse effects on economic development and social stability [7-9]. Therefore, the rapid acquisition of comprehensive tailings pond inventories is of great significance to regional effective resource utilization and mine safety monitoring.

Traditional ground surveys of tailings ponds are costly and inefficient $[4,10,11]$. Remote sensing technology has the advantages of a wide monitoring range and low cost $[12,13]$, which provides more opportunities to monitor tailings ponds. However, the shapes, dimensions, backgrounds and tones of different tailings ponds vary greatly in 
remote images covering huge geospatial areas because of the influence of mine types, geography and climate conditions on tailings ponds in different regions [14]. These characteristics also make the extraction of tailings pond margins a challenge.

Due to the abundant spectral and texture features of tailings ponds in remote sensing images, these features are typically utilized to design remote sensing indices to extract tailings [15-17]. Ma et al. (2018) developed an ultra-low-grade iron-related objects index from Landsat 8 OLI (Operational Land Imager) images and then extracted the tailings ponds based on the entropy difference between tailings ponds and stopes [16]. Using Landsat 8 data, Hao et al. (2019) developed an all-band tailing index, a modified normalized difference tailing index and a normalized difference tailings index to build a tailing extraction model for extracting the tailings [17]. However, the smaller tailings ponds were difficult to extract because of the low spatial resolution of remote sensing images.

High spatial-resolution (HSR) remote sensing images can reflect the land surface comprehensively [18]. Some scholars used visual interpretation to extract tailings ponds by HSR remote sensing images [19-21]. However, visual interpretation is time-consuming and laborious when extracting tailings ponds in large areas. Using Landsat 7and SPOT (Système Probatoire d'Observation de la Terre) 5 fusion images, Mezned et al. (2016) mapped mine tailings by a linear spectral unmixing method. Based on the spatial combination of objects [22], Liu et al. (2019) extracted four main tailings pond structures, including starter dams, embankments, deposited beaches and water bodies using GaoFen-2 images [14]. However, the studies mentioned above were limited to the mine area or a few tailings ponds within the mine area. Due to excessive variation in tone, shape and dimension between tailings ponds, the methods are difficult to apply on a large area. In addition, tailings ponds are more sparsely distributed than other objects [23], making extraction of tailings ponds in a large area more difficult and time-consuming. Therefore, the quick and accurate extraction of tailings pond margins still faces challenges when using remote sensing images at large spatial areas.

Deep learning can obtain higher dimensional and abstract features of the images than traditional methods [24] and it has been proven to be a powerful technology for remote sensing image processing [24-26]. In recent years, deep learning has been widely used in land cover classification [25], scene classification [27] and ground target extraction [28,29]. The current target detection methods based on deep learning can be divided into two categories: region-based methods and regression-based methods. Region-based methods, such as Faster R-CNN (region-based convolutional neural networks) [30], achieve high precision for target detection. Nevertheless, the detection speed is slow, while the regression-based methods (e.g., SSD (Single Shot MultiBox Detector) [31] and YOLO (You Only Look Once) [32]) achieve a relatively fast detection speed. The deep learning techniques provide a new idea for extracting tailings pond margins from HSR remote sensing images in large spatial areas, which is one of the main issues in this study. In addition, the lack of public tailings pond datasets and lack of clear explanation of the abstract features extracted limit the development of tailings pond extraction by using deep learning.

To address the aforementioned limitations on extracting tailings pond margins, we produced a tailings pond dataset containing multiple tailings ponds and proposed a deep learning-based framework to quickly and accurately extract tailings pond margins from HSR remote sensing images in large spatial areas. First, the tailings ponds were located by YOLOv4 model. Then, a random forest model was applied to the detected regions to extract tailings pond margins. Finally, a morphological processing method was used to obtain the final tailings pond margins. Taking Tongling city as the study area, we extracted tailings pond margins from the HSR remote sensing image based on the proposed method.

\section{Methodology}

The flowchart of the proposed methodology in this study is illustrated in Figure 1. The methodology attempts to extract tailings pond margins using the proposed framework. The framework can be summarized by the following steps: (1) creating a tailings pond dataset 
based on the characteristics of the tailings ponds in HSR remote sensing images; (2) training the YOLOv4 model by the tailings pond dataset to obtain the tailings pond target areas; (3) building a random forest model combining the spectral and texture features to extract the initial tailings ponds based on the acquired target areas; and (4) using a morphological processing method to extract the final tailings ponds.

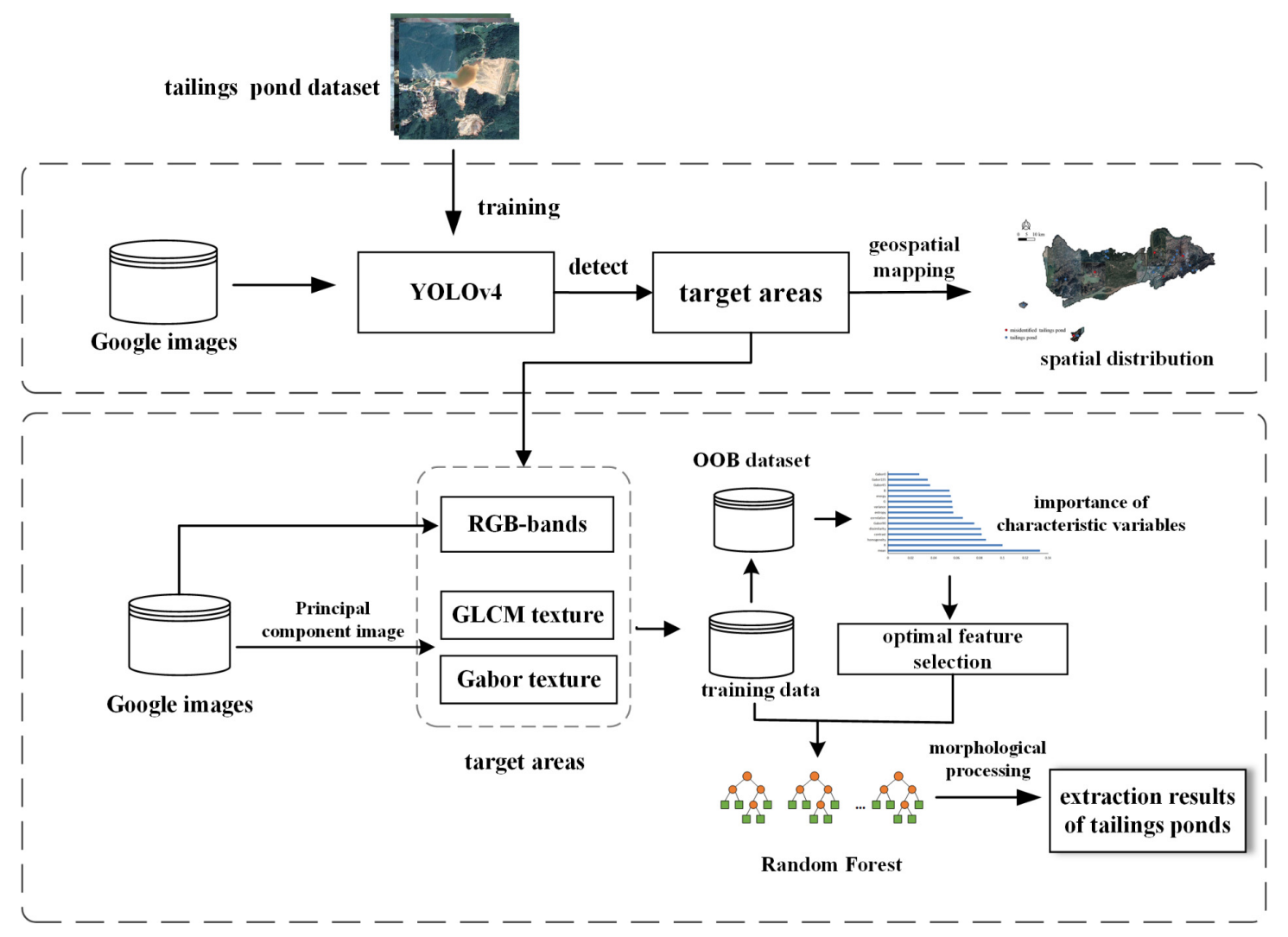

Figure 1. The flowchart of tailings pond extraction combining You Only Look Once (YOLO)v4 and random forest.

\subsection{Detection of Tailings Pond Regions}

To quickly extract the tailings pond margins in a large area, this study first detected the tailings pond regions. We have applied the YOLOv4 to detect tailings pond regions in the proposed framework. YOLOv4 achieves the best balance of accuracy and speed [33]. The characteristic empowers YOLOv4 to detect tailings ponds quickly in a large area.

The YOLOv4 algorithm was proposed by Bochkovskiy in 2020 [33]. The architecture of YOLOv4 consists of CSPDarknet53, spatial pyramid pooling (SPP), path aggregation network (PANet) and YOLOv3 head [33]. The CSPDarknet53 is used as the backbone network to extract the features of the images. The SPP block is used to increase the receptive field [34] and the PANet is adopted to merge the extracted features [35]. When scaling the input image to a size of $416 \times 416$, the three scales of feature maps are obtained after sampling five times by CSPDarkNet53. The last feature map is disposed with $5 \times 5$, $9 \times 9$ and $13 \times 13$ maximum pooling operations and then the results are merged to obtain a $13 \times 13$ feature map. When processing the acquired $13 \times 13$ feature map and the first two $26 \times 26$ and $52 \times 52$ feature maps by the modified PANet structure, three scale predictive feature maps are obtained. Finally, by performing regression and classification operations on the predicted feature maps, the boundary coordinates and confidence of tailings pond are obtained. The main network structure is shown in Figure 2. 


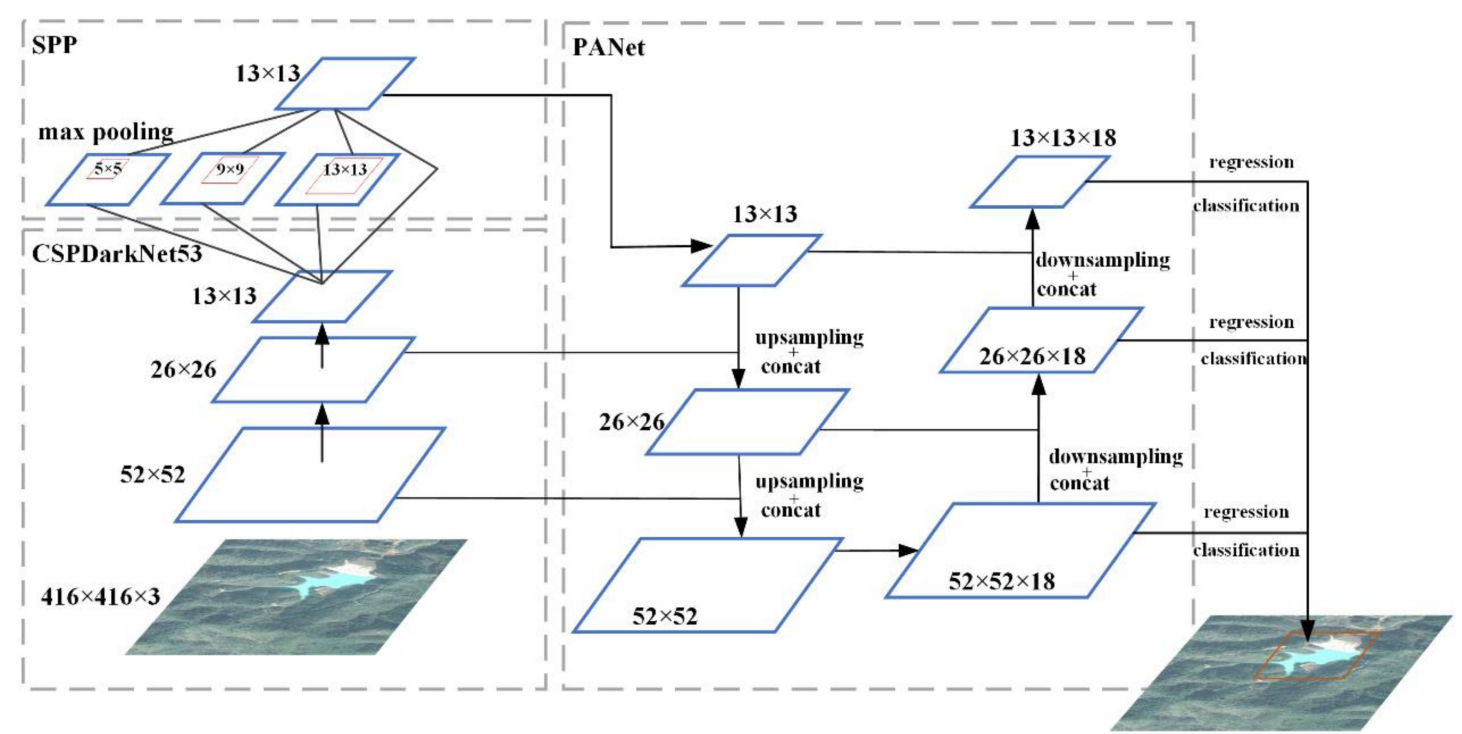

Figure 2. The main structure diagram of YOLOv4 [36].

\subsection{Extraction of Tailings Ponds}

After tailings ponds detection, the correctly detected regions were further processed to extract the precise margins of tailings ponds using random forest in our framework.

\subsubsection{Feature Extraction of Tailings Ponds}

The commonly used methods for feature extraction are divided into two categories: traditional feature extraction and deep learning extraction [25,36]. Although deep learning can provide a large number of robust features, it has the disadvantage of being difficult to interpret the extracted features [37]. To extract tailings pond features and further obtain the importance of different features, traditional feature extraction method was performed in this study. The spectral and texture features of HSR remote sensing images are able to reflect the inner components and tonal variation of ground components [38]. When two different objects have the same spectral features, they could be distinguished by their texture features [39]. Therefore, the spectral and texture features were utilized to construct a feature set for tailings pond extraction in our framework.

The RGB bands of HSR remote sensing images were selected as the spectral feature variables. Principal component analysis (PCA) was performed on the images before extracting texture features. Based on the first principal component images, the commonly used gray-level cooccurrence matrix (GLCM) and Gabor filter were utilized to obtain the texture features. We used the GLCM to extract the statistical features of the images and the Gabor filter to extract the structural features. GLCM is one of the best known texture feature extraction methods providing a good description of the image texture [40]. However, GLCM features are less accurate in the region of class boundaries [41]. Gabor filters have been proved to be successful in the field of remote sensing images [42,43]. The features extracted by Gabor filters are more accurate in the boundary regions [41]. It has been demonstrated that the combination of these two texture features can improve the accuracy of image classification $[39,41,44]$. The GLCM was used to calculate texture measurements in four directions $\left(0^{\circ}, 45^{\circ}, 90^{\circ}, 135^{\circ}\right)$ and the average of the corresponding measurement values in all directions was used as the final texture feature. Eight gray texture features (i.e., mean, variance, homogeneity, contrast, dissimilarity, entropy, second moment and correlation) were calculated with a $7 \times 7$ window size. Additionally, the Gabor filter was used to extract Gabor texture features in four directions $\left(0^{\circ}, 45^{\circ}, 90^{\circ}, 135^{\circ}\right)$ and the wavelength was set to 2 . The selected feature types and variables are shown in Table 1. 
Table 1. Feature variables used to extract the margins of tailings ponds.

\begin{tabular}{cc}
\hline Feature Type & Feature Variable \\
\hline Spectral band & $\mathrm{R}, \mathrm{G}, \mathrm{B}$ \\
Texture feature & GLCM: mean, variance, homogeneity, contrast, dissimilarity, \\
entropy, second moment, correlation \\
Gabor: Gabor0 ${ }^{\circ}$, Gabor $45^{\circ}$, Gabor $90^{\circ}$, Gabor $135^{\circ}$
\end{tabular}

\subsubsection{Random Forest Classification}

In addition to the multiple features, a robust classification algorithm is also important for extracting the tailings pond margins. Our framework utilized the random forest algorithm, which has achieved good results in the field of automatic extraction of remote sensing information [45], to further extract the tailings pond margins.

Random forest is a fusion algorithm based on decision tree classifiers with fast computational efficiency, high classification accuracy and can deal with high-dimensional features [46]. The random forest algorithm establishes corresponding decision tree models for training sets extracted from the original training dataset through the bootstrap method. The pixels are classified by taking the most popular voted class from all the forest decision trees [47]. Two-thirds of the sampled training data are used to build the classifier. The other third of the sampled training data is used to calculate the out-of-bag (OOB) error, which can evaluate the performance of random forests [48,49].

This study created a classification dataset containing tailings ponds and other objects to yield an optimal random forest model by choosing the features. Based on the importance ranking of 15 features, the features were grouped into 1 to 15 feature sets to train the random forest model. We select the feature set that has the highest OOB accuracy as the optimal feature. Finally, the chosen optimal features were used to train the random forest model for extracting tailings ponds.

\subsection{Morphological Processing}

The initial extracted image may exist some extra holes and small areas and they should be excluded before we obtain the final results. We performed morphological processing to obtain the final images.

First, we performed the hole filling operation on the initial extracted tailings pond images. A pixel point was found in the extracted tailings pond image to expand with structural elements and then constrained it with the complement of the tailings pond image. We kept repeating the expansion and constraint operation until the graph did not change and then intersected the original image to get the tailings pond image [50]. Next, we removed tiny interference. The connected regions are first extracted from the image processed in the previous step. We counted the number of pixels in each connected region. If the number was less than a given constant, the region was removed [51]. Finally, the final extracted image of the tailings pond was obtained.

\subsection{Accuracy Evaluation}

To evaluate the performance of our framework, we evaluated the detection task and final mapped results of the framework, respectively.

\subsubsection{Tailings Ponds Detection Accuracy Evaluation}

Precision, recall and mean average precision (mAP) were used in our framework to evaluate the performance of the YOLOv4 model. The formulas are as follows:

$$
\begin{gathered}
\text { Precision }=\frac{T P}{T P+F P} \\
\text { Recall }=\frac{T P}{T P+F N},
\end{gathered}
$$


where TP represents the number of tailings ponds that were correctly identified, FP represents the number of tailings ponds that were mistakenly identified and FN represents the number of tailings ponds that were not identified. The mAP value is the mean area under the precision-recall curve for all categories [52].

\subsubsection{Final Results Accuracy Evaluation}

After random forest classification and morphological processing, the obtained map of the entire study area was evaluated using the stratified sampling strategy proposed by Olofsson et al. [53,54]. We stratified by two classes: tailings ponds and other objects. The calculations of the total number of sample units and stratified sample sizes were performed [53]. The stratified random sampling was conducted by calculated sample sizes. We used the higher resolution google image and field information to determine the final reference labels. Finally, the omission and commission errors of the tailings ponds and the overall accuracy of the map were calculated based on the error matrix.

\section{Results}

\subsection{Study Area and Data}

Tongling is located in south-central Anhui Province, China. It consists of three municipal districts and one county, covering approximately 3008 square kilometers (Figure 3). Located in the copper-iron metallogenic belt of the Yangtze River, Tongling is rich in mineral resources. There are more than 30 kinds of rare metal minerals in the territory. Copper, gold, pyrite and limestone for cement are four dominant minerals [55]. However, many tailings generated after mining and smelting are stored in tailings ponds, which have become the primary source of heavy metal pollution in the environmental system of Tongling [56].

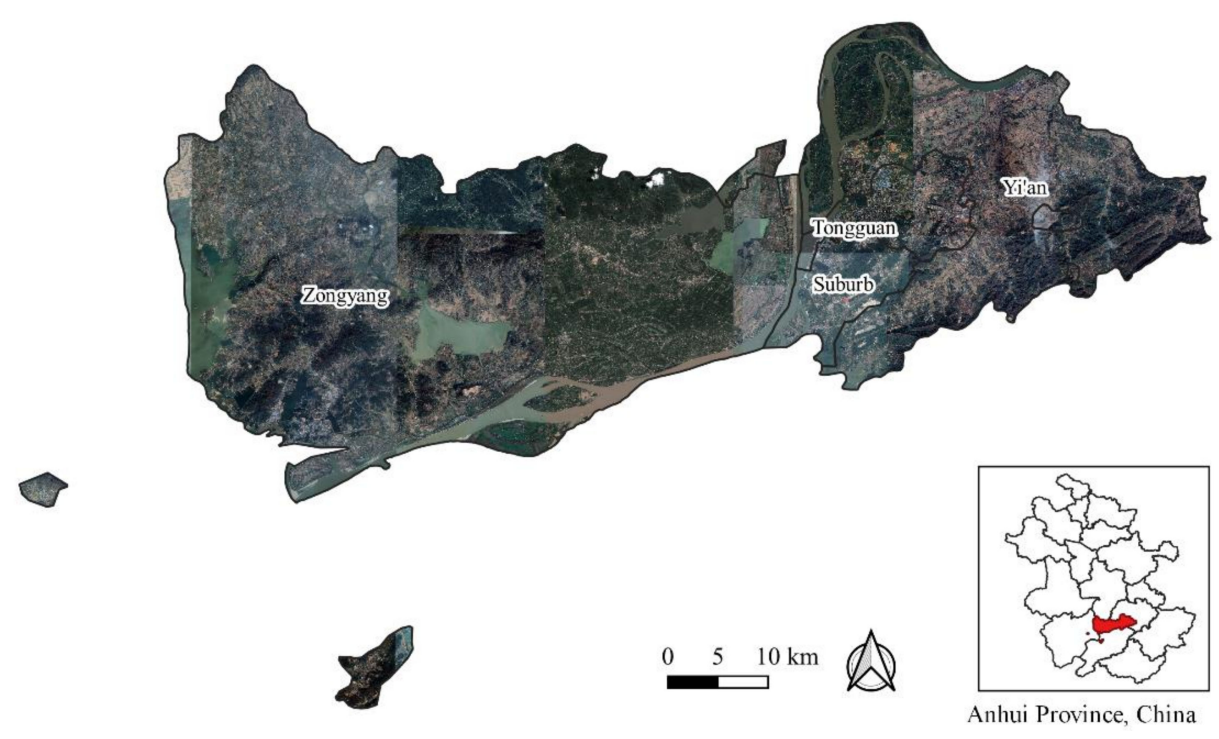

Figure 3. Study area: Tongling city, Anhui Province.

To detect tailings ponds based on deep learning, a HSR remote sensing image dataset of tailings ponds was produced. The data used in the tailings pond dataset were $2.05 \mathrm{~m}$ resolution Google images of Tongling and other cities (e.g., Ma'anshan, Chizhou, Lu'an, Huangshi), which contain tailings ponds. Considering the spatial heterogeneity of tailings ponds, samples were made by selecting various types of tailings ponds as far as possible to avoid serious omission in tailings pond results. As a preparation, we combined the color and morphological characteristics of the tailings ponds in HSR remote sensing images for visual interpretation. The remote sensing images were sliced into $500 \times 500$ pixels and then we annotated the tailings ponds in the images with the location information of the target 
category. Due to the complexity of HSR remote sensing image scenes, negative samples (e.g., reservoirs, mining areas) were added to reduce interference.

The initial dataset contained 352 positive samples and 430 negative samples. A total of 516 annotations were included in the initial dataset. However, deep learning requires a large number of samples to prevent overfitting during training and data augmentation is usually adopted [57]. The widely used data augmentation methods, including rotation, Gaussian noise, flipping and brightness variation, were adopted to increase the diversity of the samples. For image rotation and flipping, we have performed 90, 180 and 270 degrees rotations and horizontal and vertical flip operations on the images. For brightness variation, the parameters for adjusting the brightness were set to 0.5 and 1.2 and the bias was set to 10. The noise was added to the original images as well. we added Gaussian noise with a variance of 0.1 to the original images.

A positive sample was generated to 9 new images while we removed a small number of poor quality samples. To keep the number of positive and negative samples similar, we rotated, flipped and added noise to generate 7 new images for each negative sample. Via the above operations, the final tailings pond dataset contains 6150 images, including 3140 positive samples and 3010 negative samples. A total of 4610 annotations were included in the final dataset. Figure 4 shows samples in the tailings pond dataset.
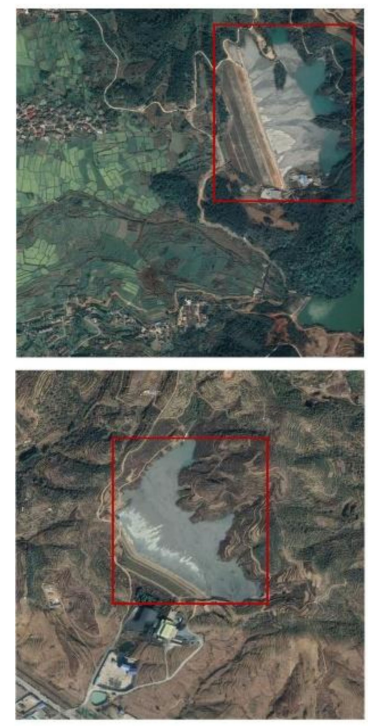

(a)
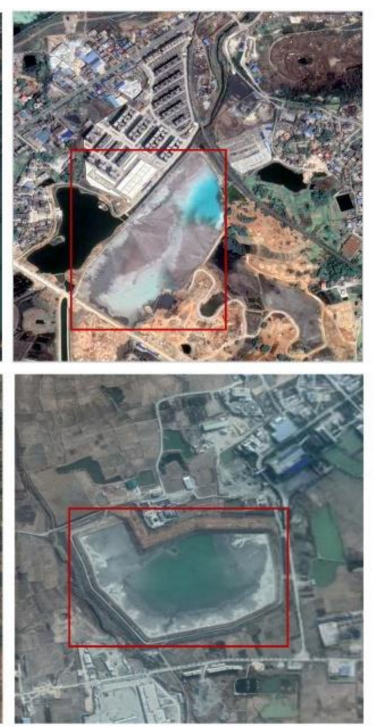

(b)
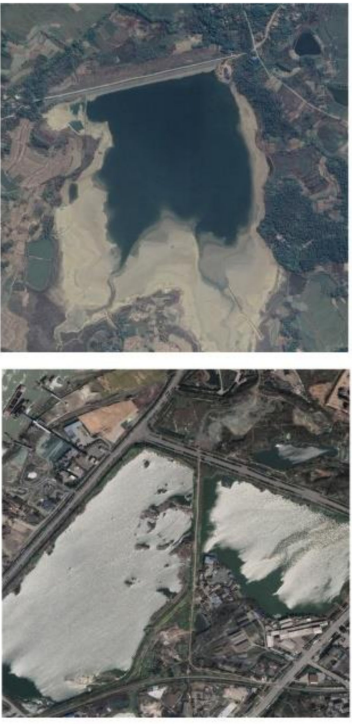

(c)
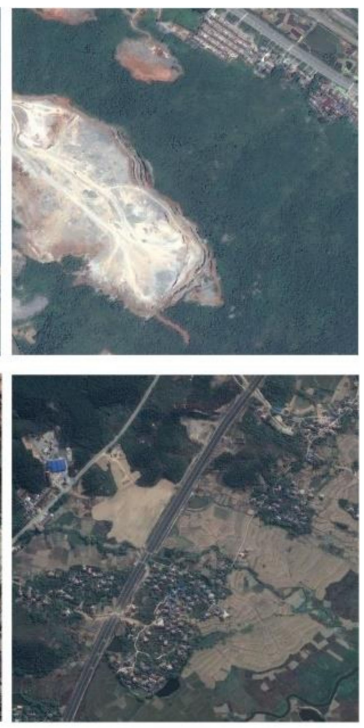

(d)

Figure 4. The samples in the tailings pond dataset. (a) and (b) are examples of tailings ponds with different shapes and colors. (c) and (d) are examples of negative samples.

\subsection{Detection of Tailings Ponds Based on YOLOv4}

The tailings pond dataset was randomly divided into a training set and testing set at a ratio of 8:2. The YOLOv4 algorithm was trained for 300 iterations and the batch size was set to 4 . The default hyperparameters which could obtain the great performance in YOLO [58], were applied with a learning rate of 0.01 , the momentum of 0.937 and weight decay of 0.0005 .

A multiscale training strategy was adopted to enhance the robustness of images of different sizes. During the training process, the image size was randomly transformed into multiples of 32 and the image transformation range was set as $320 \times 320 \sim 608 \times 608$ [59]. After approximately 250 iterations of the model, the training loss tended to be stable. For the confidence threshold of 0.5 , the model performed the best $\mathrm{mAP}$ in the testing set when the image size was $448 \times 448$ and it was selected as the final tailings ponds detector. As shown in Table 2, the precision is $99.6 \%$, the recall rate is $89.9 \%$ and the $\mathrm{mAP}$ is $89.7 \%$. 
The results showed that the YOLOv4 algorithm can detect tailings ponds accurately and provide a reasonable basis for further extraction of tailings ponds.

Table 2. Detection accuracy evaluation results of tailings ponds based on YOLOv4.

\begin{tabular}{cccc}
\hline Confidence & Precision & Recall & mAP \\
\hline 0.5 & $99.6 \%$ & $89.9 \%$ & $89.7 \%$ \\
\hline
\end{tabular}

Taking the Tongling as a study area, the final tailings ponds detector was used to detect the tailings ponds from HSR remote sensing image. The images of Tongling were sliced into $500 \times 500$ pixel size and a total of 2745 images were obtained. We obtained 26 tailings ponds as the truth value by visual interpretation. For the confidence threshold of 0.5 , 31 features were detected as suspected tailings ponds. The tailings ponds obtained by visual interpretation were all included in the detected regions. The distribution of targets obtained by geospatial mapping was illustrated in Figure 5. The detected targets were verified by visual interpretation and the information provided by the tailings pond managers. Five false positive targets were detected by YOLOv4, mainly including reservoirs, waste rock piles and bare ground. Examples of incorrectly identified targets were shown in (d) and (e). (d) was a bare ground with similar shape to the tailings pond. (e) was a reservoir with a similar structure to the tailings pond, including water bodies and beaches. The color of the water body was similar to the color of the tailing pond wastewater, showing a similar brightness to that of the tailing pond. Inactive tailings ponds contained very little water. The bare grounds and the exposed land in small reservoirs were similar in shape, texture to the tailings in inactive tailings ponds. These similar properties made the YOLOv4 model prone to errors in prediction. Objects that were incorrectly detected as tailings ponds were excluded from the final results. A total of 26 tailings ponds were finally recognized in Tongling. The correctly detected tailings ponds were served as the basis for further extracting tailing ponds.

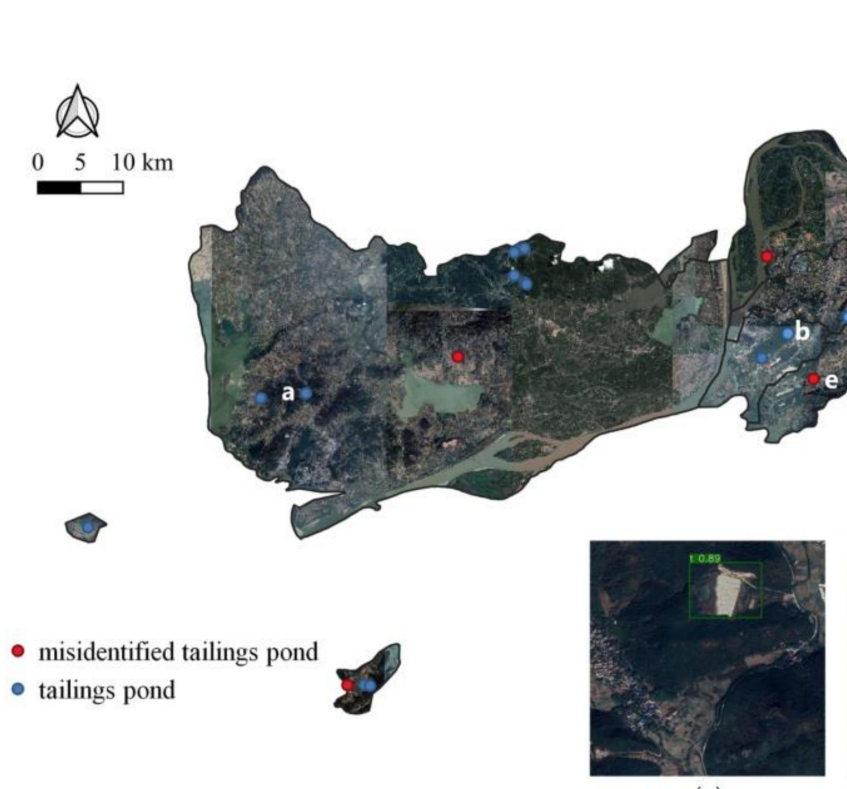

(a)
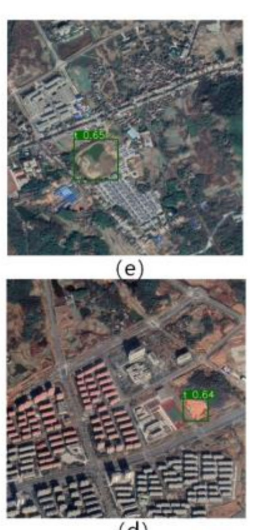

(d)

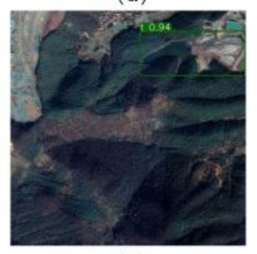

(c)

Figure 5. The spatial distribution results of tailings ponds in Tongling based on the trained YOLOv4. The blue points are the correctly identified results and the red points are the misidentified results. (a), (b) and (c) are examples of correctly identified tailings ponds; (d) and (e) are examples of misidentified tailings ponds. 


\subsection{The Extraction of Tailings Ponds in Tongling}

Considering that the characteristics of tailings ponds vary greatly in different regions, we performed the selection of tailings classification samples in the correctly detected regions. This study created a training dataset containing 28,704 pixels for two classes. The number of decision trees was set to 500, which is a frequently used value when using the random forest classifier on remote sensing data [60].

\subsubsection{Selection of Optimal Features Based on Random Forest}

The importance ranking of the 15 features is shown in Figure 6. The variation in OOB accuracy (\%) concerning the number of features used during random forest based extraction of tailings ponds is demonstrated in Figure 7. The OOB accuracy started to increase with the increase in the input features until reaching an optimum, after which it began to decrease. The OOB accuracy achieved $91.38 \%$ by using all the features. In contrast, the OOB accuracy achieved $92.03 \%$ by using 12 features. Therefore, the top 12 features were selected as the feature variables to obtain an optimal random forest model.

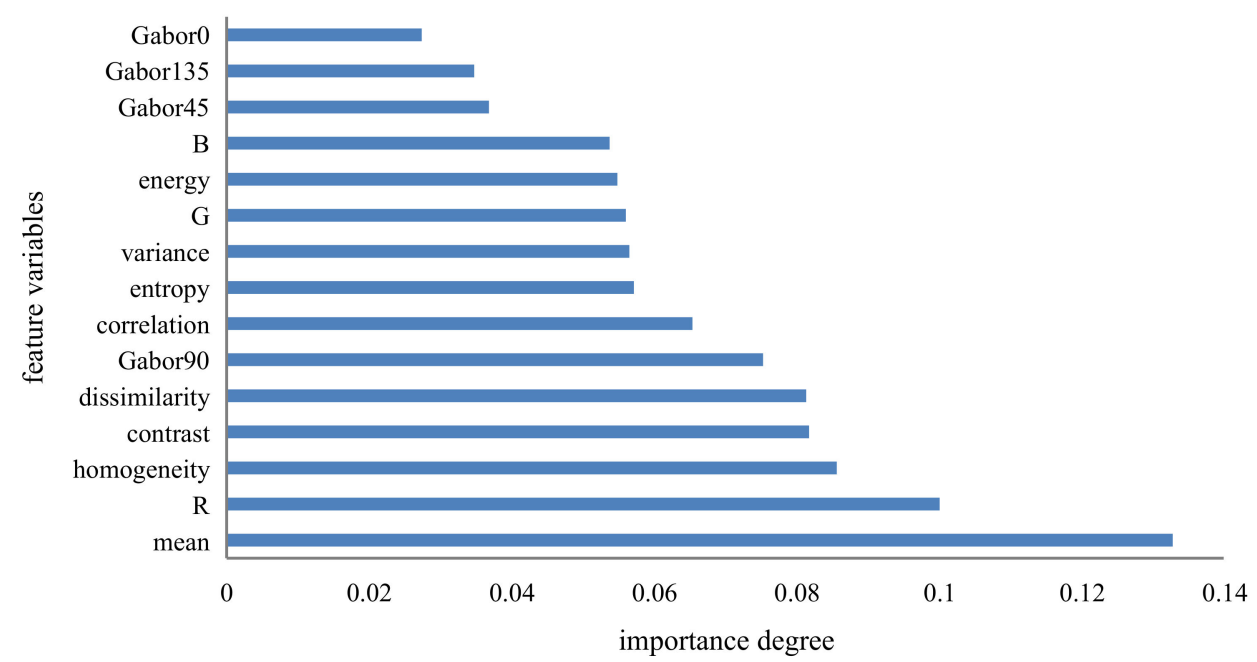

Figure 6. The importance of feature variables obtained through the random forest model.

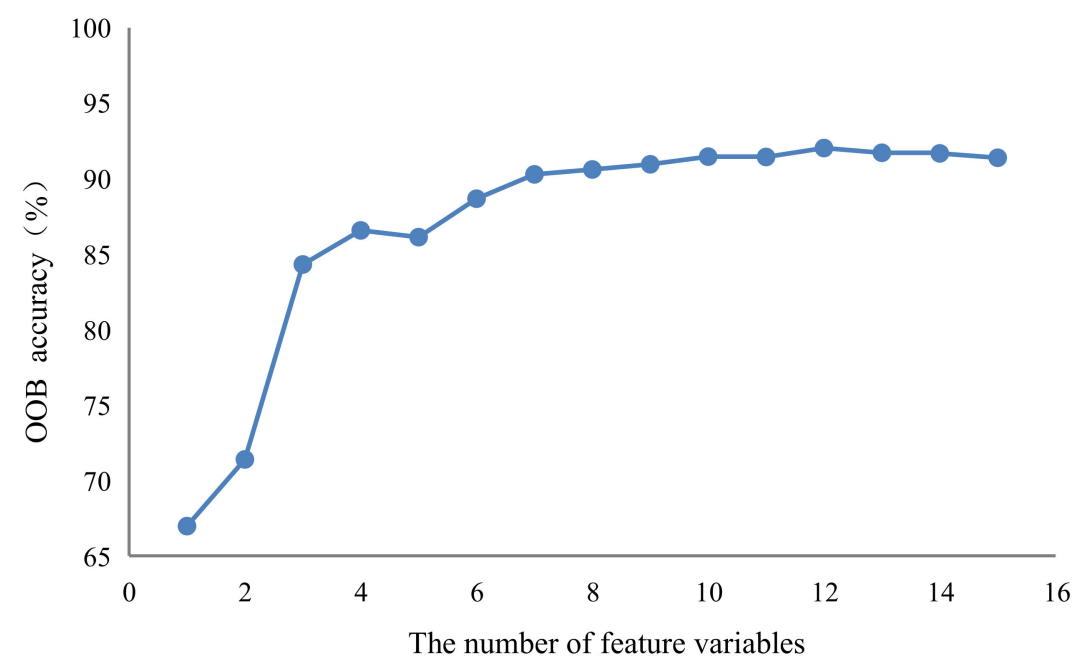

Figure 7. The relationship between the number of feature variables and out-of-bag (OOB) accuracy.

\subsubsection{Extraction Results of Tailings Ponds}

This study selected the optimal random forest model to extract tailings ponds. Due to the existence of internal holes and broken areas in the extracted tailings ponds (Figure 8b), 
we used hole filling and small area removal methods in morphological processing to extract final tailings ponds. Examples of tailings pond extraction results are shown in Figure 8.

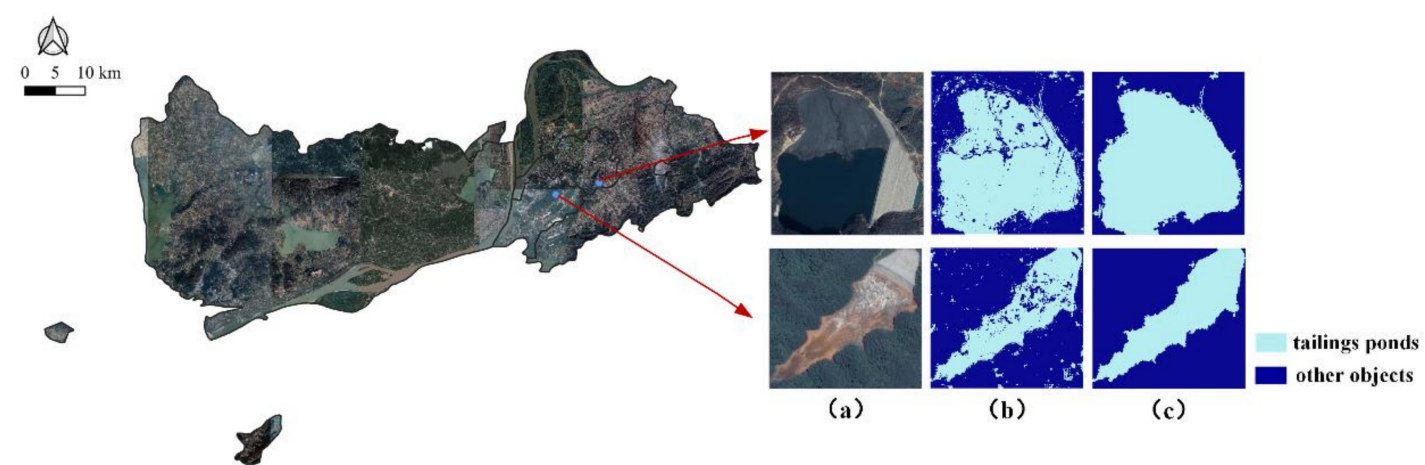

Figure 8. Examples of tailings pond extraction results based on the proposed method. (a) are the target areas of the tailings ponds identified by YOLOv4, (b) are the extraction results by random forest model and (c) are the results of $b$ after morphological processing.

\subsubsection{Final Map Accuracy Assessment}

The obtained map of the entire study area was evaluated using the stratified sampling strategy. A total of 90,000 sample units (pixels) was finally obtained. The two strata of the tailings ponds and other objects were allocated 3200 and 86,800, respectively. The error matrix based on the estimated proportions of areas was shown in Table 3. The overall accuracy was $99.98 \%$ and the omission and commission errors of the tailings ponds were $18.75 \%$ and $7.15 \%$, respectively. The results indicated that the map of the entire study area was obtained with great performance.

Table 3. The error matrix based on the estimated proportions of areas $W_{i}$ is the proportion of area mapped as class i.

\begin{tabular}{ccccc}
\hline Class & Tailings Ponds & Other Objects & $\begin{array}{c}\text { Map Area } \\
\text { (Pixel) }\end{array}$ & Total $\left(\boldsymbol{W}_{\boldsymbol{i}}\right)$ \\
\hline Tailings ponds & 0.00052 & 0.00004 & 337,070 & 0.00056 \\
Other objects & 0.00012 & 0.99932 & $604,802,377$ & 0.99944 \\
Total & 0.00064 & 0.99936 & $605,139,447$ & 1 \\
\hline
\end{tabular}

\subsection{Model Comparison Results}

To prove the proposed method's efficiency in extracting tailings ponds in a large area, a conventional random forest pixel classification method for tailings ponds extraction was also used in the experiments for comparison. In the whole study area, substantial disk resources are required for storage and calculation after fusing multiple texture features. Therefore, we chose Tongshan town in the study area (approximately 0.017 times the size of Tongling) to compare the extraction time of different methods.

As shown in Table 4, the extraction time of the proposed method in Tongshan town was $17.8 \mathrm{~s}$ and the extraction time in the whole study area was $378.5 \mathrm{~s}$. However, the extraction time of random forest classification in Tongshan town was $844.7 \mathrm{~s}$. Combined with the morphology of tailing ponds in HSR remote sensing images, the margins of tailing ponds detected by YOLOv4 were outlined to verify the extraction results. Although the tailings ponds were well extracted by plain random forest (Figure 9a), the misclassification of other regions was obvious. In the results of (b) and (c), the misclassified regions were greatly reduced and better extraction results were obtained. Overall, compared with only using random forest classification, the proposed method was more efficient and effective in extracting tailings ponds. 
Table 4. Comparison of extraction time of tailings ponds based on different methods.

\begin{tabular}{ccc}
\hline Comparative Experiment & Extraction Time in Tongshan Town & Extraction Time in the Study Area \\
\hline YOLOv4 + random forest & 17.8 seconds & 378.5 seconds \\
Only random forest & 844.7 seconds & Approximately 13 hours \\
\hline
\end{tabular}

(a)

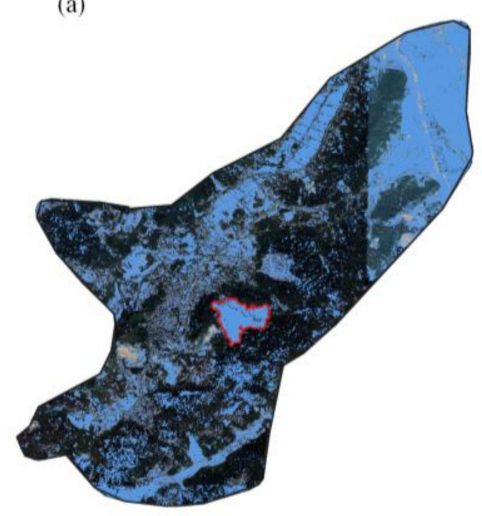

(b)

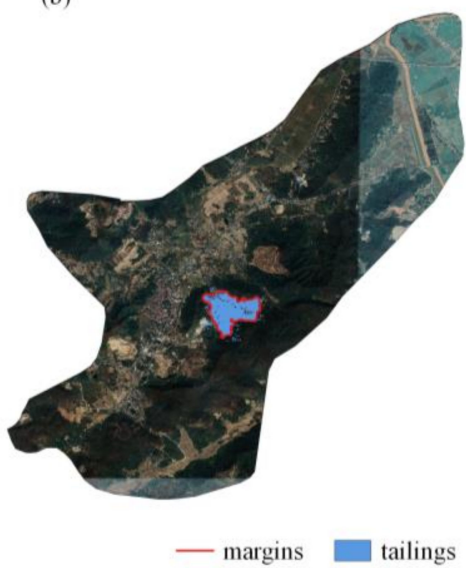

(c)

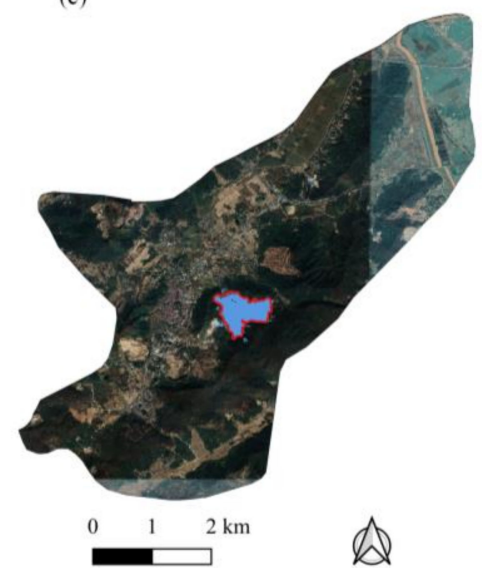

Figure 9. The extraction results of tailings ponds in Tongshan town based on different methods. (a) was the extraction result by plain random forest, (b) was the extraction result by YOLOv4+random forest model and (c) was the result of the whole framework (YOLOv4 + ranodm forest + morphological processing).

\section{Discussion}

In previous studies, few researchers used HSR remote sensing images to extract tailings pond margins in large spatial areas. This study proposed a framework that combined YOLOv4 and the random forest algorithm to extract tailings pond margins. First, the YOLOv4 model was trained with a tailings pond dataset to obtain the tailings pond locations. On this basis, the optimal random forest model combined with spectral and texture features was utilized to extract initial tailings ponds on the correctly detected regions. Finally, hole filling and small area removal methods were performed on the initial results to obtain the final extraction results of tailings ponds.

This study first combined deep learning with random forest to extract tailings pond margins. Our proposed framework can be applied to the areas where satellite images are available. The proposed framework can quickly locate tailings ponds and extract them with great extraction performance, significantly reducing the time for visual interpretation of remote sensing images. A map that contains the tailings ponds and the areas classified as another type could also be further obtained. Besides, we have produced a tailings pond dataset using visual interpretation, which contains various types of tailings ponds and has proved to be valid. The dataset could provide data support for relevant research on tailings ponds.

Tailings ponds are only a small part of the total area. Considering the actual demand of mineral resources inventory, the image area we need to extract is usually large (e.g., city, province, etc.). When the margins of tailings ponds need to obtain in a large area, the performance of extraction and the saving of computing resources have to be taken into account. Therefore, the YOLOv4 model was used to detect the tailings ponds, which is the key step of the proposed framework. The YOLOv4 model would address the issue of the time-consuming of tailings pond extraction in HSR remote sensing images and accurately locate to tailings pond regions. Based on the tailings pond dataset, with a confidence threshold of 0.5 , we obtained high accuracy (precision $=99.6 \%$, recall $=89.9 \%$, $\mathrm{mAP}=89.7 \%$ ). Experiment results showed that the YOLOv4 model in our framework could effectively detect tailings pond locations from a large spatial HSR remote sensing image and provide a reasonable basis for the further extraction of tailings ponds. 
Compared with the current study, the area of Tongling (approximately $3008 \mathrm{~km}^{2}$ ) is relatively large. Applying the proposed method to extract tailings ponds in Tongling, we obtained 26 correctly detected regions and 5 wrongly detected regions. In the actual application, there are inevitably some objects, such as reservoirs and bare ground that have similar characteristics to tailing ponds, causing YOLOv4 detection errors. This is the reason for the discrepancy between model accuracy and the results in the actual application. Based on correctly detected regions, the optimal random forest model and morphological processing were used to further extract tailings ponds. Finally, we obtained a map of the entire study area containing tailings ponds and other objects. The obtained map was evaluated based on a stratified sampling strategy. The omission and commission errors of the tailings ponds were $18.75 \%$ and $7.15 \%$, respectively, with an overall accuracy of $99.98 \%$. The results showed that our framework could obtain great performance in extracting the tailings ponds. Since the YOLOv4 model excluded a large number of other objects, the areas outside the target regions and the apparently misidentified target regions were classified as other objects when we mapped the entire study area results. The target regions were small relative to the whole area and other objects misclassified as tailings ponds only existed in the target regions, resulting in a small probability of misclassification of other objects and high overall accuracy.

In addition, the total extraction time of the proposed framework was $378.5 \mathrm{~s}$. The computational time of the proposed framework was mainly affected by the number of image pixels and the longest time-consuming step was the detection calculation of the YOLOv4 model. The extraction efficiency and effectiveness of the proposed method were much better than only using random forest classification. The reason for the results is that we first used the YOLOv4 model to accurately locate the tailings ponds, which significantly reduced the time to classify irrelevant features by the random forest model and improved the extraction effect of tailings ponds from another aspect. In addition, in the previous studies, the Mask R-CNN model has been used in the task of boundary extraction [61]. However, the Mask R-CNN mainly focuses on the accuracy of the model and considers the speed less [62]. In practice, large scale and high-frequency monitoring are needed. The algorithm would be very time consuming when applied to large HSR remote sensing images (e.g., city, province, etc.) [63]. In contrast, our framework combining the advantages of YOLOv4's speed to make it more suitable for practical applications. The combination of the YOLOv4 and the random forest enabled our entire framework to take speed into account while considering accuracy.

Furthermore, by conducting random forest feature optimization, we found that the mean texture feature and R-band feature were dominant in the extraction. The optimal features that were selected included spectral bands, GLCM texture and Gabor texture. The results mean that the appropriate combination of spectral and texture features played an important role in improving tailings pond extraction accuracy. In addition, the highest $\mathrm{OOB}$ accuracy of $92.03 \%$ was obtained when the top 12 feature variables were selected. Then, the OOB accuracy tended to decline (Figure 7). The reason resulting this phenomenon may be that there is a specific correlation among multiple features adopted in this study. The participation of all features in classification would lead to information redundancy, thus reducing classification accuracy [64].

Although our framework obtained great performance, some details of our framework may be enhanced in the future. First, Tongling is a relatively large region and the applicability of our framework would be verified in city clusters in our future studies. Second, due to the complexity and diversity of tailings ponds, which are influenced by the natural conditions and policies of the regions in which they are located, the original tailings pond dataset in the study was small and we used a data augmentation approach to expand the dataset. The detection effect of the model may be affected when the study area changes. In the future, it is necessary to improve the completeness of the dataset by collecting more samples from other cities and to explore the impact of different data enhancement methods to further enhance the robustness of the detection model. Additionally, despite the high 
overall accuracy of the obtained map in our study, there are still omission and commission errors of tailings ponds. The pixel classification we used inevitably reduced the extraction effect of the tailings ponds. The object-oriented classification method could be considered in the future. Finally, tailings ponds have strong spatial heterogeneity and the characteristics of tailings ponds in different regions vary greatly. Therefore, the extraction model of random forest needs to be adjusted to further enhance the robustness by combining the characteristics of local tailings ponds. Moreover, in addition to extracting tailings ponds, future studies may consider adding hyperspectral remote sensing data and DEM data to study the scope of infiltration pollution of tailings ponds and conduct an environmental risk assessment.

\section{Conclusions}

This study proposed a framework that combines YOLOv4 with the random forest algorithm to extract tailings pond margins from large spatial HSR remote sensing images and created an opened source tailings pond dataset. Taking Tongling as the study area and based on our dataset, our proposed framework achieved high accuracy in extracting tailings pond locations (precision $=99.6 \%$, recall $=89.9 \%, \mathrm{mAP}=89.7 \%$ ). Based on an optimal random forest model and morphological processing, tailings pond margins were further extracted and the map of the entire study area was obtained with an overall accuracy of $99.98 \%$. Our framework is more efficient in contrast to the random forest algorithm. The proposed framework could extract various tailings pond locations and margins with high accuracy and speed from a large spatial HSR remote sensing image. In addition, we found that the appropriate combination of spectral and texture features played an essential role in improving tailings pond extraction accuracy. This study can provide an effective inventory method for tailings ponds in government departments and a useful reference for mine safety and environmental monitoring.

Author Contributions: Conceptualization, J.L. and Y.Y.; Data curation, Y.H.; Formal analysis, S.R., D.D., Q.G. and L.T.; Funding acquisition, Y.Y.; Methodology, Y.Y.; Project administration, J.L. and Y.Y.; Validation, Y.H.; Writing —original draft, J.L. and Y.H. All authors have read and agreed to the published version of the manuscript.

Funding: This work was supported by the National Key R\&D Program of China (Grant No. 2019YFB2102903), the National Natural Science Foundation of China (Grant No. 41801306 and 41671408).

Institutional Review Board Statement: Not applicable.

Informed Consent Statement: Not applicable.

Data Availability Statement: The tailings pond datasets and the code that support this study are available with the identifiers at the private link: https:/ figshare.com/s/ee2da2d0bc6e27a2a582 (accessed on 15 February 2021).

Acknowledgments: We really appreciate the editors and the three anonymous reviewers for their useful comments and suggestions. We would like to thank the No.321 Geological Team, Bureau of Geological and Mineral Exploration of Anhui Province for the data support.

Conflicts of Interest: The authors declare no conflict of interest.

\section{References}

1. $\quad$ BG, L. Mine Wastes: Characterization, Treatment and Environmental Impacts, 3rd ed.; Springer: Berlin/Heidelberg, Germany, 2010.

2. Komnitsas, K.; Kontopoulos, A.; Lazar, I.; Cambridge, M. Risk assessment and proposed remedial actions in coastal tailings disposal sites in Romania. Miner. Eng. 1998, 11, 1179-1190. [CrossRef]

3. Nasategay, F.F.U. Detection and Monitoring of Tailings Dam Surface Erosion Using UAV and Machine Learning; University of Nevada: Reno, NV, USA, 2020.

4. Xiao, R.; Lv, J.; Fu, Z.; Wenming, S.; Wencheng, X.; Yuanli, S.; Fei, C.; Qianxiang, X. The application of remote sensing in the environmental risk monitoring of tailings pond in Zhangjiakou city, China. Remote Sens. Technol. Appl. 2014, 29, 100-105. 
5. Tang, L.; Liu, X.; Wang, X.; Liu, S.; Deng, H. Statistical analysis of tailings ponds in China. J. Geochem. Explor. 2020, $216,106579$. [CrossRef]

6. Kossoff, D.; Dubbin, W.E.; Alfredsson, M.; Edwards, S.J.; Macklin, M.G.; Hudson-Edwards, K.A. Mine tailings dams: Characteristics, failure, environmental impacts and remediation. Appl. Geochem. 2014, 51, 229-245. [CrossRef]

7. Mei, G. Quantitative assessment method study based on weakness theory of dam failure risks in tailings dam. Proc. Eng. 2011, 26, 1827-1834.

8. Lyu, Z.; Chai, J.; Xu, Z.; Qin, Y.; Cao, J. A comprehensive review on reasons for tailings dam failures based on case history. Adv. Civil Eng. 2019, 9, 1-18. [CrossRef]

9. Yu, D.; Tang, L.; Chen, C. Three-dimensional numerical simulation of mud flow from a tailings dam failure across complex terrain. Nat. Hazard. Earth Sys. 2020, 20, 727-741. [CrossRef]

10. Zabcic, N.; Rivard, B.; Ong, C.; Müller, A. Using airborne hyperspectral data to characterize the surface $\mathrm{pH}$ and mineralogy of pyrite mine tailings. Int. J. Appl. Earth Obs. 2014, 32, 152-162. [CrossRef]

11. Che, D.; Liang, A.; Li, X.; Ma, B. Remote sensing assessment of safety risk of iron tailings pond based on runoff coefficient. Sensors 2018, 18, 4373. [CrossRef]

12. Lawley, V.; Lewis, M.; Clarke, K.; Ostendorf, B. Site-based and remote sensing methods for monitoring indicators of vegetation condition: An Australian review. Ecol. Indic. 2016, 60, 1273-1283. [CrossRef]

13. Tan, Q.; Shao, Y. Application of remote sensing technology to environmental pollution monitoring. Remote Sens. Technol. Appl. 2012, 15, 246-251.

14. Liu, K.; Liu, R.; Liu, Y. A Tailings Pond Identification Method Based on Spatial Combination of Objects. IEEE J. Sel. Top. Appl. Earth Observ. Remote Sens. 2019, 12, 2707-2717. [CrossRef]

15. Schimmer, R. A remote sensing and GIS method for detecting land surface areas covered by copper mill tailings. In Proceedings of the Pecora, Denver, CO, USA, 18-20 November 2008.

16. Ma, B.; Yuteng, C.; Song, Z.; Xuexin, L. Remote Sensing Extraction Method of Tailings Ponds in Ultra-Low-Grade Iron Mining Area Based on Spectral Characteristics and Texture Entropy. Entropy 2018, 20, 345. [CrossRef]

17. Hao, L.; Zhang, Z.; Yang, X. Mine tailing extraction indexes and model using remote-sensing images in southeast Hubei Province. Environ. Earth Sci. 2019, 78, 493. [CrossRef]

18. Zhang, X.; Xiao, P.; Feng, X.; Yuan, M. Separate segmentation of multi-temporal high-resolution remote sensing images for object-based change detection in urban area. Remote Sens. Environ. 2017, 201, 243-255. [CrossRef]

19. Hao, L.; Zhang, Z.; He, W.X.; Chen, T. Tailings reservoir recognition factors of the high resolution remote sensing image in Southeastern Hubei. Remote Sens. Land Resour. 2012, 24, 154-158.

20. Gao, Y.; Chu, Y.; Liang, W. Remote sensing monitoring and analysis of tailings ponds in the ore concentration area of Heilongjiang Province. Remote Sens. Land Resour. 2015, 27, 160-163.

21. Zhou, Y.; Wang, X.; Yao, W.; Yang, J. Remote sensing investigation and environment impact analysis of tailing ponds in Shandong province. Geol. Surv. China 2017, 4, 88-92.

22. Mezned, N.; Mechrgui, N.; Abdeljaouad, S. Mine wastes environmental impact mapping using Landsat ETM+ and SPOT 5 data fusion in the North of Tunisia. J. Indian Soc. Remote 2016, 44, 451-455. [CrossRef]

23. Li, Q.M.; Zhang, H.; Li, G. Comparative analysis on safety management of tailings ponds in China and Canada. China Min. Mag. 2017, 26, 21-24.

24. Kussul, N.; Lavreniuk, M.; Skakun, S.; Shelestov, A. Deep learning classification of land cover and crop types using remote sensing data. IEEE Geosci. Remote Sens. 2017, 14, 778-782. [CrossRef]

25. Zhang, L.; Xia, G.; Wu, T.; Lin, L.; Tai, X.C. Deep learning for remote sensing image understanding. J. Sens. 2016, 501, 173691. [CrossRef]

26. Ball, J.E.; Anderson, D.T.; Chan, C.S. Comprehensive survey of deep learning in remote sensing: Theories, tools and challenges for the community. J. Appl. Remote Sens. 2017, 11, 42609. [CrossRef]

27. Zou, Q.; Ni, L.; Zhang, T.; Wang, Q. Deep learning based feature selection for remote sensing scene classification. IEEE Geosci. Remote Sens. 2015, 12, 2321-2325. [CrossRef]

28. Alshehhi, R.; Marpu, P.R.; Woon, W.L.; Mura, M.D. Simultaneous extraction of roads and buildings in remote sensing imagery with convolutional neural networks. ISPRS J. Photogramm. Remote Sens. 2017, 130, 139-149. [CrossRef]

29. Cao, Y.; Niu, X.; Dou, Y. Region-based convolutional neural networks for object detection in very high resolution remote sensing images. In Proceedings of the 12th International Conference on Natural Computation, Fuzzy Systems and Knowledge Discovery (ICNC-FSKD), Changsha, China, 13-15 August 2016.

30. Ren, S.; He, K.; Girshick, R.; Sun, J. Faster r-cnn: Towards real-time object detection with region proposal networks. In Proceedings of the Advances in Neural Information Processing Systems, Montreal, QC, Canada, 7-12 December 2015.

31. Liu, W.; Anguelov, D.; Erhan, D.; Szegedy, C.; Reed, S.; Fu, C.; Berg, A.C. Ssd: Single shot multibox detector. In Proceedings of the 14th European Conference on Computer Vision, Amsterdam, The Netherlands, 8-16 October 2016.

32. Redmon, J.; Divvala, S.; Girshick, R.; Farhadi, A. You only look once: Unified, real-time object detection. In Proceedings of the IEEE Conference on Computer Vision and Pattern Recognition (CVPR), Las Vegas, NV, USA, 27-30 June 2016. 
33. Bochkovskiy, A.; Wang, C.; Liao, H.M. YOLOv4: Optimal Speed and Accuracy of Object Detection. arXiv 2020, arXiv:2004.10934. (preprint).

34. Cheng, Z.; Zhang, F. Flower End-to-End Detection Based on YOLOv4 Using a Mobile Device. Wirel. Commun. Mob. Comput. 2020, 2, 1-9. [CrossRef]

35. Liu, S.; Qi, L.; Qin, H.; Shi, J.; Jia, J. Path aggregation network for instance segmentation. In Proceedings of the Conference on Computer Vision and Pattern Recognition (CVPR), Salt Lake City, UT, USA, 18-23 June 2018; pp. 8759-8768.

36. Seifi Majdar, R.; Ghassemian, H. A probabilistic SVM approach for hyperspectral image classification using spectral and texture features. Int. J. Remote Sens. 2017, 38, 4265-4284. [CrossRef]

37. Zhang, Q.; Zhu, S. Visual interpretability for deep learning: A survey. Front. Inf. Technol. Electron. Eng. 2018, 19, 27-39. [CrossRef]

38. Liu, X.; He, J.; Yao, Y.; Zhang, J.; Liang, H.; Wang, H.; Hong, Y. Classifying urban land use by integrating remote sensing and social media data. Int. J. Geogr. Inf. Sci. 2017, 31, 1675-1696. [CrossRef]

39. Tan, K.; Zhang, Y.; Wang, X.; Chen, Y. Object-based change detection using multiple classifiers and multi-scale uncertainty analysis. Remote Sens. 2019, 11, 359. [CrossRef]

40. Hall-Beyer, M. Practical guidelines for choosing GLCM textures to use in landscape classification tasks over a range of moderate spatial scales. Int. J. Remote Sens. 2017, 38, 1312-1338. [CrossRef]

41. Mirzapour, F.; Ghassemian, H. Fast GLCM and Gabor filters for texture classification of very high resolution remote sensing images. Int. J. Inf. Commun. Technol. Res. 2015, 7, 21-30.

42. Hao, M.; Shi, W.; Ye, Y.; Zhang, H.; Deng, K. A novel change detection approach for VHR remote sensing images by integrating multi-scale features. Int. J. Remote Sens. 2019, 40, 4910-4933. [CrossRef]

43. Chen, L.; Zhu, Q.; Xie, X.; Hu, H.; Zeng, H. Road extraction from VHR remote-sensing imagery via object segmentation constrained by Gabor features. ISPRS Int. J. Geo Inf. 2018, 7, 362. [CrossRef]

44. Imani, M.; Ghassemian, H. GLCM, Gabor and morphology profiles fusion for hyperspectral image classification. In Proceedings of the 24th Iranian Conference on Electrical Engineering (ICEE), Shiraz University, Shiraz, Iran, 10-12 May 2016.

45. Teluguntla, P.; Thenkabail, P.S.; Oliphant, A.; Xiong, J.; Gumma, M.K.; Congalton, R.G.; Yadav, K.; Huete, A. A 30-m landsatderived cropland extent product of Australia and China using random forest machine learning algorithm on Google Earth Engine cloud computing platform. ISPRS J. Photogramm. 2018, 144, 325-340. [CrossRef]

46. Breiman, L. Random forests. Mach. Learn. 2001, 45, 5-32. [CrossRef]

47. Pal, M. Random forest classifier for remote sensing classification. Int. J. Remote Sens. 2005, 26, 217-222. [CrossRef]

48. Beyer, F.; Jurasinski, G.; Couwenberg, J.; Grenzdörffer, G. Multisensor data to derive peatland vegetation communities using a fixed-wing unmanned aerial vehicle. Int. J. Remote Sens. 2019, 40, 9103-9125. [CrossRef]

49. Yao, Y.; Liu, X.; Li, X.; Liu, P.; Hong, Y.; Zhang, Y.; Mai, K. Simulating urban land-use changes at a large scale by integrating dynamic land parcel subdivision and vector-based cellular automata. Int. J. Geogr. Inf. Sci. 2017, 31, 2452-2479. [CrossRef]

50. Rafael, G.C.; Richard, W.E.; Steven, E.L. Digital Image Processing, 3rd ed.; Prentice Hall: Upper Saddle River, NJ, USA, 2007.

51. An, Z.; Shi, Z. Scene learning for cloud detection on remote-sensing images. IEEE J. Sel. Top. Appl. Earth Observ. Remote Sens. 2015, 8, 4206-4222. [CrossRef]

52. Everingham, M.; Van Gool, L.; Williams, C.K.; Winn, J.; Zisserman, A. The pascal visual object classes (voc) challenge. Int. J. Comput. Vision 2010, 88, 303-338. [CrossRef]

53. Olofsson, P.; Foody, G.M.; Herold, M.; Stehman, S.V.; Woodcock, C.E.; Wulder, M.A. Good practices for estimating area and assessing accuracy of land change. Remote Sens. Environ. 2014, 148, 42-57. [CrossRef]

54. Olofsson, P.; Foody, G.M.; Stehman, S.V.; Woodcock, C.E. Making better use of accuracy data in land change studies: Estimating accuracy and area and quantifying uncertainty using stratified estimation. Remote Sens. Environ. 2013, 129, 122-131. [CrossRef]

55. Wang, B.; Ye, X.; Cheng, C. Comprehensive Approach to Solve the Eco-environmental Problem in the Mining Area of Tongling City, Anhui Province. Resour. Environ. Yangtze Basin 2004, 13, 494-498.

56. Wang, J.; Xu, X.; Chen, F. Assessment of the heavy metals pollution in the reclaimed soil of Linchong tailings reservoir, Tongling. J. Hefei Univ. Technol. (Nat. Sci.) 2005, 2, 142-145.

57. Perez, L.; Wang, J. The effectiveness of data augmentation in image classification using deep learning. arXiv 2017, arXiv:1712.04621. (preprint).

58. Mandal, V.; Mussah, A.R.; Adu-Gyamfi, Y. Deep Learning Frameworks for Pavement Distress Classification: A Comparative Analysis. arXiv 2020, arXiv:2010.10681. (preprint).

59. Possatti, L.C.; Guidolini, R.; Cardoso, V.B.; Berriel, R.F.; Paixão, T.M.; Badue, C.; De Souza, A.F.; Oliveira-Santos, T. Traffic light recognition using deep learning and prior maps for autonomous cars. In Proceedings of the International Joint Conference on Neural Networks, Budapest, Hungary, 14-19 July, 2019.

60. Belgiu, M.; Drăguț, L. Random forest in remote sensing: A review of applications and future directions. ISPRS J. Photogramm. 2016, 114, 24-31. [CrossRef]

61. He, K.; Gkioxari, G.; Dollár, P.; Girshick, R. Mask r-cnn. In Proceedings of the IEEE International Conference on Computer Vision (ICCV), Venice, Italy, 22-29 October 2017.

62. Zhang, Q.; Chang, X.; Bian, S.B. Vehicle-damage-detection segmentation algorithm based on improved mask RCNN. IEEE Access 2020, 8, 6997-7004. [CrossRef] 
63. Xue, Y.; Jia, F.; Cai, X.; Shadabfare, M. Semantic Segmentation of Shield Tunnel Leakage with Combining SSD and FCN. Intell. Syst. Appl. 2020, 1250, 53-61.

64. Georganos, S.; Grippa, T.; Vanhuysse, S.; Lennert, M.; Shimoni, M.; Kalogirou, S.; Wolff, E. Less is more: Optimizing classification performance through feature selection in a very-high-resolution remote sensing object-based urban application. Gisci. Remote Sens. 2018, 55, 221-242. [CrossRef] 